\title{
BMJ Open Risk of cardiac and non-cardiac adverse events in community-dwelling older patients with atrial fibrillation: a prospective cohort study in the Netherlands
}

Sander van Doorn, Annerien Tavenier, Frans H Rutten, Arno W Hoes, Karel G M Moons, Geert-Jan Geersing

To cite: van Doorn S, Tavenier A, Rutten FH, et al. Risk of cardiac and non-cardiac adverse events in community-dwelling older patients with atrial fibrillation: a prospective cohort study in the Netherlands. BMJ Open 2018;8:e21681. doi:10.1136/ bmjopen-2018-021681

- Prepublication history and additional material for this paper are available online. To view these files, please visit the journal online (http://dx.doi. org/10.1136/bmjopen-2018021681).

Accepted 30 July 2018

Check for updates

(C) Author(s) (or their employer(s)) 2018. Re-use permitted under CC BY-NC. No commercial re-use. See rights and permissions. Published by BMJ.

Julius Center for Health Sciences and Primary care, University Medical Center Utrecht, Utrecht, The Netherlands

Correspondence to Dr Sander van Doorn; s.vandoorn@umcutrecht.nl

\section{ABSTRACT}

Objectives Patients with atrial fibrillation (AF) are at increased risk of many adverse events, notably stroke. To prevent all adverse outcomes, integrated AF care is advocated though the potential domain for such multidisciplinary management is still unclear. Therefore, insight in the systemic nature of AF and identifying patients at risk of adverse events after oral anticoagulation is needed. The aim of this study is to first describe the risk of hospitalisation and mortality in community-dwelling older patients with AF using anticoagulants, and second to assess the association between traditional cardiac risk factors and these outcomes.

Design A prospective cohort.

Setting General practice.

Participants 2068 patients with AF using oral anticoagulants.

Outcome measures We calculated incidence rates (IRs) of ischaemic stroke, bleeding, hospitalisations and mortality, and compared risk factors using Cox regression between those with and without an adverse event, both for cardiac and non-cardiac causes.

Results During a median follow-up of 2.7 (IQR 2.2-3.0) years, the IR per 100 person-years was 22.1 for hospitalisations and 6.7 for mortality. Non-cardiac events outnumbered cardiac events (IRs 15.7 vs 7.6 per 100 person-years for hospitalisation, $p<0.001$ and 5.0 vs $1.7, p<0.001$ for mortality). As a comparison, the IRs for stroke and major bleeding were 1.7 and 0.8 per 100 person years, respectively. In multivariate models, high age, heart failure and vascular disease were independently associated with all-cause hospitalisation and - in addition to diabetes, previous stroke and renal disease-for allcause mortality.

Conclusions In anticoagulated community-dwelling patients with AF, stroke risk is effectively reduced and thus fairly low, whereas risks of hospitalisation and mortality remain high, importantly mainly for non-cardiac causes. Notably high age, heart failure and vascular disease are predictive for such outcomes and may be of value in identifying high-risk patients in the future.

Trial registration number NTR3741.
Strengths and limitations of this study

- We could study a large prospective cohort of representative community-dwelling patients with atrial fibrillation (AF) using anticoagulation.

- Data on hospitalisation, mortality and risk factors were manually verified using all information in the electronic patient file.

In the Cox proportional hazard analyses, we have not taken competing risks into account.

- Our study does not include a control group of patients without AF.

\section{INTRODUCTION}

Atrial fibrillation (AF) is the most common cardiac arrhythmia with a prevalence of $1 \%-2 \%$ in the general population, increasing to over $10 \%$ in those aged 80 years and older. $^{12}$ Studies both in European countries, including the Netherlands ${ }^{3}$ and the $\mathrm{UK}^{4}{ }^{4}$ as well as in the USA, ${ }^{5}$ consistently expect the number of patients with AF to double in the coming decades due to the ageing population and better detection. Since the famous Framingham paper in the early $90 \mathrm{~s}$, AF is considered a major risk factor for (cardioembolic) stroke. ${ }^{6}$ Thus, since then, prevention of stroke using oral anticoagulants is the mainstay of (chronic) treatment of AF, first with vitamin $\mathrm{K}$ antagonists (VKA) and more recently with direct oral anticoagulants (DOAC).

It is increasingly being recognised, however, that $\mathrm{AF}$ is not merely an arrhythmia and risk factor for stroke, but instead is a complex interplay of clinical conditions and pathophysiological alterations. Processes like inflammation, hypercoagulability, endothelial dysfunction and (finally) fibrosis accelerate the progression and burden of $\mathrm{AF}$, stroke risk and related comorbidities such as 
(in particular) heart failure. ${ }^{7-10}$ This puts patients with $\mathrm{AF}$ at increased risk of stroke and many other adverse events. Hospital admissions are of longer duration and occur more frequently in patients with AF compared with those without. ${ }^{1-13}$ Finally, the risk of renal failure and death is higher. ${ }^{14}$ Clearly, this systemic nature of AF calls for interventions beyond anticoagulation alone that aim to prevent all relevant adverse events, as in fact is also advocated in the latest guideline by the European Society of Cardiology. ${ }^{15}$

While those who will benefit from anticoagulation to prevent stroke may be identified using traditional stroke prediction models such as CHA2DS2-VASc, ${ }^{16}$ insight into the other risks in community-dwelling patients with $\mathrm{AF}$ is largely missing. Therefore, to evaluate this systemic nature of $\mathrm{AF}$ and to characterise patients at risk of adverse events beyond stroke alone, the aim of our study was twofold. First, we wanted to describe the risks of cardiac and non-cardiac hospitalisations and mortality in a cohort of older community-dwelling patients with $\mathrm{AF}$ already treated with oral anticoagulants, and second-in order to tailor future patients' management-we wanted to explore the association between these outcomes and well-known risk factors in anticoagulated patients with AF.

\section{METHODS}

\section{Study population}

The cohort of anticoagulated community-dwelling patients with AF was part of the CAFe trial (Cost-effectiveness of balancing stroke and bleeding risk using CHA2DS2-VASc in primary care patients with Atrial Fibrillation, www.trialregister. nl NTR3741, registered Pre-results on Dec 6th 2012), a large prospective cluster-randomised trial evaluating automated decision-support on the treatment and outcome of patients with $\mathrm{AF}$ in general practice in the Netherlands.

General practices in the Utrecht region, the Netherlands, were invited to participate in the CAFe study. From February 2013 to September 2014, a planned total of 38 practices enrolled in the study. At baseline, an automated search in the registers of these 38 practices identified all older patients diagnosed with $\mathrm{AF}$ based on the International Classification of Primary Care (ICPC) ${ }^{17}$ code K78 'atrial fibrillation/flutter'. In addition, files were scrutinised containing ICPC codes for cardiac arrhythmias (K97 'paroxysmal tachycardia' and K80 'ectopic beats/extra-systoles'), as well as files of patients receiving a recent prescription of antiarrhythmic drugs (ie, amiodarone, sotalol, digoxin and flecainide) and/or oral anticoagulants (ie, VKA and DOAC). Subsequently, for every identified patient, the electronic patient file was manually screened by the researchers for correctness of AF diagnosis (ie, whether it was indeed confirmed by ECG). Furthermore, to avoid misclassification, all available information including diagnostic test results, out-of-hours office reports and specialists' letters (that are regularly received by general practitioners in the Netherlands) was used to assess the correctness of the important stroke risk predictors (ie, diagnoses included in the CHA2DS2-VASc stroke prediction model either established in general practice or in hospitals). For the current study, all patients (already) using oral anticoagulants at baseline were included. Sex and age (in years) were extracted from the electronic patient file.

Finally, Anatomic Therapeutic Chemical (ATC) codes of all drug prescriptions, including oral anticoagulants and platelet inhibitors, in the year previous to study inclusion were collected. Drugs were categorised into pharmacological subgroups based on second-level or third-level ATC code (see online supplementary I).

\section{Outcomes}

Patients were followed up for at least 2 years. At follow-up, all electronic patient files of the included patients with AF were again manually scrutinised. Using all available information in the electronic patient file, the following outcome variables were recorded in the 2 years after baseline data collection:

- Ischaemic stroke, transient ischaemic attack (TIA) and/or thromboembolism.

- Bleeding.

- Hospitalisation (yes/no), further classified as:

- Cardiac hospitalisation.

- Non-cardiac hospitalisation, including diagnosis at hospital admission.

- Mortality (yes/no), further classified as:

- Cardiac mortality.

- Non-cardiac mortality, including cause of death.

Stroke was defined as a focal neurological deficit of sudden onset lasting $>24$ hours not attributable to other identifiably causes. TIA was defined as a focal neurological deficit of sudden onset lasting $<24$ hours. Thromboembolism was defined as peripheral embolism or pulmonary embolism. Peripheral embolism was defined as a sudden occlusion of an artery to an extremity or a visceral organ outside the brain, heart, eyes and lungs, not attributable to concomitant atherosclerosis or other aetiology. Pulmonary embolism was defined as radiographic confirmation of a pulmonary arterial occlusion.

Bleeding was defined as major bleeding and/or clinically relevant non-major bleeding, according to the definitions of the International Society on Thrombosis and Haemostasis. ${ }^{18}$

As multiple reasons may ultimately have led to hospitalisation, we decided on the primary cause for hospitalisation based on all available information in the electronic file, including hospital referral letters and discharge letters.

Patients were censored at the time of the adverse event, at the time of death, at the time of lost to follow-up or at the end of the follow-up, whichever came first.

\section{Patient and public involvement}

No patients or public were involved in the design, conduct or dissemination of this study. 


\section{Data analyses}

In every patient, a risk factor was considered present if the electronic patient file contained a respective diagnosis, whereas a risk factor was considered absent when the electronic patient file did not contain a respective diagnosis code. As such, there was (strictly speaking) no missing data for the available ICPC codes as we deemed the respective risk factor absent in case it was not recorded in the electronic patient files.

Incidence rates (IR) for each outcome of interest (cardiac, non-cardiac and all-cause hospitalisation; and cardiac, non-cardiac and all-cause mortality) were expressed as the number of events per 100 person-years of follow-up. We used univariable Cox proportional hazard models to assess the association between individual well-known stroke risk factors and hospitalisation and mortality. We subsequently used a multivariable Cox model to assess the adjusted HRs, with associated 95\% CIs.

All analyses were performed in R V.3.3.2 with the package rms V.5.1-0.

\section{RESULTS}

\section{Patient characteristics}

In the total number of 38 participating general practices, we identified 2355 patients with a confirmed diagnosis of AF, of which a total $2068(88 \%)$ already used anticoagulation at baseline, and they were included in the current study. The median age in this anticoagulated cohort was 78 (IQR 69-84) years and $1255(61 \%)$ were 75 years or older, and $51 \%$ of the patients were male. Of the common cardiovascular risk factors, hypertension was most prevalent $(63 \%)$. See table 1 for the patient characteristics. At baseline, $97.1 \%$ of patients used a VKA, and 2.9\% used a DOAC (all dabigatran). In the 12 months before baseline, a total of $769(37.2 \%)$ patients used any of the antiarrhythmic drugs recommended for rhythm control by the European Society of Cardiology

\begin{tabular}{lc}
\hline $\begin{array}{l}\text { Table } 1 \text { Characteristics of } 2068 \text { community-dwelling } \\
\text { patients with AF using anticoagulants }\end{array}$ \\
\hline \multicolumn{2}{l}{$\mathbf{n = 2 0 6 8 , \mathbf { n } ( \% )}$} \\
\hline Median age (IQR) & $78(69-84)$ \\
Age $<65$ years & $283(13.7)$ \\
Age 65-74years & $530(25.6)$ \\
Age $\geq 75$ years & $1255(60.7)$ \\
Female sex & $1017(49.2)$ \\
Heart failure & $419(20.3)$ \\
Hypertension & $1296(62.7)$ \\
Diabetes & $503(24.3)$ \\
Stroke & $368(17.8)$ \\
Renal disorder & $331(16)$ \\
Vascular disease & $571(27.6)$ \\
\hline
\end{tabular}

$\mathrm{AF}$, atrial fibrillation. and available in the Netherlands (amiodarone in 131 $(6.3 \%)$, flecainide in $217(10.5 \%)$, propafenone in 7 $(0.3 \%)$, and sotalol in $478(23.1 \%)$ patients, respectively). Online supplementary I shows the subgroups of most frequently prescribed drugs in the year before the start of the study.

Total follow-up was 5133 person-years (median follow-up 2.7 years, IQR 2.2-3.0 years). In total, 197 (9.5\%) patients left the study before completing follow-up, after a median follow-up of 1.5 years (IQR 0.7-2.2 years). Compared with patients with a follow-up of at least 2 years, they were older (median age 84.0 vs 77.0 years, $\mathrm{p}<0.001$ ), more often female $(59 \%$ vs $48 \%, \mathrm{p}=0.003)$ and more often suffered heart failure $(26 \%$ vs $20 \%, \mathrm{p}=0.03)$ and renal disorder (23\% vs $15 \%, \mathrm{p}=0.004)$. In total, 66 of these 197 patients left the study after moving to residential care (eg, nursing homes), the remaining 131 patients moved out of the area.

During follow-up, 45 (2.2\%) patients underwent arrhythmia procedures, predominantly catheter ablation.

\section{Stroke and bleeding}

Despite anticoagulant treatment, during follow-up stroke occurred in 87 patients with an IR of 1.73 per 100 personyears. Major bleeding and clinically relevant non-major bleeding occurred with an IR of 0.9 and 2.7 per 100 person-years, respectively.

\section{Hospitalisation}

In total, 879 patients were admitted to the hospital at least once (IR 22.1 per 100 person-years). Hospitalisation occurred most frequently for non-cardiac causes compared with cardiac causes (IR 15.7 and 7.6 per 100 person-years, respectively, $\mathrm{p}<0.001)$. In fact, $66 \%$ of all hospitalisations was due to a non-cardiac cause. Table 2 shows the reasons for these non-cardiac hospitalisations. Infectious diseases, internal diseases, cancer and pulmonology conditions accounted for half of all admissions. About one-third was admitted to surgery or orthopaedics, 116 because of falls of which $75(65 \%)$ had traumatic fractures.

Patients admitted to the hospital during follow-up had a higher median age (79 vs 77 years, HR $1.02,95 \%$ CI 1.01 to 1.03 ) and CHA2DS2-VASc score (4 vs 3, HR 1.2, 95\% CI 1.1 to 1.2 ). Table 3 shows the traditional risk factors for those with and without admittance to the hospital. In the univariable model, the proportion of females did not differ statistically significantly (HR 1.1, 95\% CI 0.9 to 1.2). All other CHA2DS2-VASc risk factors, in addition to renal disease, were more frequently seen in those hospitalised. In the multivariable model, only high age, heart failure and vascular disease remained independently associated with hospitalisation. While previous stroke was also predictive of hospitalisation in females (adjusted OR 1.17, 95\% CI 1.05 to 1.29), additional stratified analyses by sex did not further change our results (data not shown). 
Table 2 Non-cardiac causes for hospitalisation

\begin{tabular}{|c|c|}
\hline & n (\%) \\
\hline Cancer & 72 (10.5) \\
\hline Breast & $8(11.1)$ \\
\hline Digestive tract & 24 (33.3) \\
\hline Respiratory tract & $8(11.1)$ \\
\hline Urogenital tract & $21(29.2)$ \\
\hline Neurologic al & $3(4.2)$ \\
\hline Ha ematologic al & $3(4.2)$ \\
\hline Other & $5(6.9)$ \\
\hline Infectious disease/internal disease & $232(34.0)$ \\
\hline Urogenital tract & $28 \quad(12.1)$ \\
\hline Respiratory tract & $63 \quad(27.2)$ \\
\hline Digestive tract & $16 \quad(6.9)$ \\
\hline Skin & $16(6.9)$ \\
\hline Other infectious disease & $12(5.2)$ \\
\hline Other internal disease & 97 (41.8) \\
\hline Orthopaedics & $120(17.6)$ \\
\hline Elective & $45 \quad(37.5)$ \\
\hline Fractures & 75 (62.5) \\
\hline Surgery & $119(17.4)$ \\
\hline Vascular & $27 \quad(22.7)$ \\
\hline Fall, no fractures & $41 \quad(34.5)$ \\
\hline Other & $51 \quad(42.9)$ \\
\hline Pulmonology & $36(5.3)$ \\
\hline COPD & $21 \quad(58.3)$ \\
\hline Other & 15 (41.7) \\
\hline Neurology & $40(5.9)$ \\
\hline Urology/gynaecology & $27(4.0)$ \\
\hline Other & 37 (5.4) \\
\hline
\end{tabular}

\section{Mortality}

In total 343, patients died (IR 6.7 per 100 person-years), of whom 87 patients (IR 1.7 per 100 person-years) died from a cardiac cause. In the 258 non-cardiac deaths (IR 5.0 per 100 person-years), cancer and infectious disease again were the most frequent causes.

Patients who died during follow-up were older and showed higher CHA2DS2-VASc scores than those who survived (84 vs 76 years, HR 1.09, 95\% CI 1.08 to 1.10 ; and 5 vs 3 , HR 1.4, 95\% CI 1.3 to 1.4, respectively). All risk factors were more frequently seen in those who survived, with the exception of hypertension in the univariable model, and hypertension and female sex in the multivariable model (see table 4). Results remained largely unchanged in analyses stratified by sex though the HR for diabetes was not statistically significant in females (HR $1.21,95 \%$ CI 0.85 to 1.57 ) (further data not shown).

\section{DISCUSSION}

In anticoagulated community-dwelling patients with $\mathrm{AF}$, we found a fairly low IR of stroke and bleeding. These rates indeed were far exceeded by rates of hospitalisation and mortality, importantly most often due to non-cardiac causes rather than cardiac causes. Notably, high age, heart failure and vascular disease were associated with these adverse outcomes. These findings underline the importance of $\mathrm{AF}$ as a signal of cardiorespiratory-metabolic decline and-in our view-exemplify the need for integrated care approaches.

\section{Comparison with existing literature}

Previous research found similar high rates of hospitalisation in patients with $\mathrm{AF}$ though underlying causes differed. For instance, in a Danish study also non-cardiac hospitalisation outnumbered cardiac hospitalisation 
Table 3 Risk factors for hospitalisation

\begin{tabular}{|c|c|c|c|c|}
\hline & $\begin{array}{l}\text { No hospitalisation } \\
(n=1189)\end{array}$ & $\begin{array}{l}\text { With hospitalisation } \\
(\mathrm{n}=879)\end{array}$ & $\begin{array}{l}\text { Univariate HR } \\
(95 \% \mathrm{Cl})\end{array}$ & $\begin{array}{l}\text { Multivariate HR } \\
(95 \% \mathrm{Cl}) \dagger\end{array}$ \\
\hline Median age (IQR) & 77 (68-84) & $79(72-85)$ & $1.02(1.01 \text { to } 1.03)^{\star}$ & $1.02(1.01 \text { to } 1.03)^{*}$ \\
\hline Female sex & $581(48.9)$ & $436(49.6)$ & 1.05 (0.92 to 1.18$)$ & 0.95 (0.81 to 1.09$)$ \\
\hline Heart failure & $200(16.8)$ & $219(24.9)$ & 1.57 (1.42 to 1.72$)$ & 1.37 (1.20 to 1.53$)$ \\
\hline Diabetes & 264 (22.2) & 239 (27.2) & 1.25 (1.1 to 1.4$)$ & 1.14 (0.99 to 1.29$)$ \\
\hline Stroke & $199(16.7)$ & 169 (19.2) & 1.10 (1.02 to 1.18$)$ & $1.06(0.97$ to 1.14$)$ \\
\hline Renal disease & $173(14.6)$ & $158(18)$ & 1.38 (1.21 to 1.55$)$ & $1.06(0.87$ to 1.24$)$ \\
\hline Vascular disorder & $284(23.9)$ & $287(32.7)$ & 1.45 (1.31 to 1.59$)$ & 1.28 (1.14 to 1.43$)$ \\
\hline
\end{tabular}

Numbers are counts (percentages) unless specified otherwise.

${ }^{*}$ Per 1 point/year increase.

†Adjusted for all other risk factors except the CHA2DS2-VASc score.

particularly in older patients with $\mathrm{AF}^{19}$ similar as in our study. Conversely, elderly patients in the Outcomes Registry for Better Informed Treatment of Atrial Fibrillation registry and the Euro Heart Survey were hospitalised with rates of $\sim 30 \%$ year, but in contrast mainly for cardiovascular conditions. ${ }^{11}{ }^{20}$ Finally, in primary care patients with AF in Germany, hospitalisation occurred in $18.5 \%$ of patients each year, largely comparable with our findings,${ }^{21}$ although non-cardiac causes were not further specified. In our study, we found that non-cardiac causes mostly consisted of infectious diseases and hospitalisation for oncological conditions. For mortality, similar differences were observed. For instance, patients in our cohort died mainly from non-cardiac causes, whereas in the contemporary DOAC trials ${ }^{22}$ and in the EURObservational Research Programme-Atrial Fibrillation registry in cardiology clinics ${ }^{23}$ death from a cardiac cause was most frequent with IRs around 2.1 per 100 person-years. In fact, our observed IR of 1.7 per 100 person-years for cardiac mortality was rather comparable with these estimates from the recent DOAC trials, whereas the rates for non-cardiac mortality were more than twofold higher (IR 5.0 per 100 person-years). In line with previous research, we found besides high age that heart failure and vascular disease both predicted (cardiac and non-cardiac) hospitalisation and mortality. ${ }^{411} 24$

These partly conflicting findings on the comparative risks for cardiac and non-cardiac hospitalisation and mortality in patients with $\mathrm{AF}$ across studies likely are explained by the clinical setting and patient population of each study. Where in cardiology clinic populations cardiac arrhythmia-related admissions and interventions (including, eg, ablation procedures) may (still) predominate, the older and multimorbid community-dwelling population with chronic $\mathrm{AF}$ is at risk of adverse outcomes across multiple organ systems that largely exceed cardiac events. Although not a primary aim of this study to explore, we believe these findings underpin the recognition of the

Table 4 Risk factors for mortality

\begin{tabular}{|c|c|c|c|c|}
\hline & $\begin{array}{l}\text { No mortality } \\
(n=1724)\end{array}$ & $\begin{array}{l}\text { With mortality } \\
(n=344)\end{array}$ & $\begin{array}{l}\text { Univ. HR } \\
\text { (95\% Cl) }\end{array}$ & $\begin{array}{l}\text { Multiv. HR } \\
(95 \% \mathrm{Cl}) \dagger\end{array}$ \\
\hline Median age (IQR) & $76(68-83)$ & $84(78-89)$ & $1.09(1.08 \text { to } 1.1)^{*}$ & $1.08(1.07 \text { to } 1.1)^{\star}$ \\
\hline Female sex & $828(48)$ & $189(54.9)$ & $1.29(1.08$ to 1.5$)$ & 0.92 (0.69 to 1.14$)$ \\
\hline Heart failure & $302(17.5)$ & $117(34)$ & 2.23 (2.01 to 2.45 ) & 1.4 (1.17 to 1.63$)$ \\
\hline Stroke & $274(15.9)$ & $94(27.3)$ & 1.36 (1.24 to 1.48$)$ & $1.22(1.1$ to 1.34$)$ \\
\hline Renal disease & $238(13.8)$ & $93(27)$ & 2.27 (2.03 to 2.51$)$ & 1.28 (1.03 to 1.53$)$ \\
\hline Vascular disorder & $436(25.3)$ & 135 (39.2) & 1.78 (1.56 to 2$)$ & 1.39 (1.17 to 1.62$)$ \\
\hline
\end{tabular}

Numbers are counts (percentages) unless specified otherwise.

${ }^{*}$ Per 1 point/year increase.

†Adjusted for all other risk factors except the CHA2DS2-VASc score. 
systemic nature of AF, where ageing and processes such as inflammation, hypercoagulability, endothelial dysfunction and fibrosis interact. ${ }^{11}$ Such processes may not only accelerate the progression and burden of AF itself, but also mutually reinforce the progression of other cardiorespiratory-metabolic conditions. This more or less reflects 'an engine slowly running out of fuel' ${ }^{10}$ resulting in a frailty that in our study is further illustrated by the large number of patients moving to residential care. Management of chronic AF in a community or primary healthcare setting therefore calls for additional efforts (ie, beyond stroke prevention and rhythm control) in order to improve all relevant patient outcomes. ${ }^{25}$

\section{Strengths and limitations}

Strengths of our study include the fairly large and representative prospective cohort of community-dwelling patients with AF using anticoagulation and a follow-up of over 2 years. Practices covered urban, suburban and rural areas, and patients were included irrespective of (co-) treatment by a cardiologist. We were able to evaluate all relevant well-known stroke risk factors, each obtained manually using all available information in the electronic patient file, and included both cardiovascular and non-cardiovascular outcomes.

A limitation of our study is that in our Cox proportional hazards analyses, all hospitalisations were considered equally and competing risks were not taken into account. Indeed, over $7 \%$ of patients in our study were hospitalised three times or more. These patients were slightly older to those with no or fewer hospitalisations (mean age 77.3 vs 76.3 years, $p=0.56$ ) but had a higher mean CHA2DS2-VASc score (3.2 vs 4.2, $\mathrm{p}=0.002$ ). Although we have not yet evaluated this subgroup of patients with $\mathrm{AF}$ at risk for multiple hospitalisations, their apparent frailty certainly warrants future attention. Furthermore, for some outcomes under study the statistical power was somewhat low.

In addition, although all patients were prescribed anticoagulant medication, we did not have any information on Internation Normalised Ratio (INR) values or actual treatment adherence. Some outcomes may therefore have occurred while patients were not in the so-called therapeutic INR range.

A last important limitation of our study is that, by design, our cohort study did not have a control group. We aimed to describe anticoagulated patients with $\mathrm{AF}$ at highest risk of adverse events, and as such cannot evaluate the impact on morbidity and mortality of AF itself on such events. Unfortunately, to the best of our knowledge studies comparable with ours in patients without $\mathrm{AF}$ are lacking. However, municipal registries could provide an indication of the magnitude of the risks in our study. For instance, according to the Central Bureau of Statistics in the Netherlands, the overall annual incidence of death in patients aged 65 years and older in 2016 was $4.1 \%$. Importantly, this number includes all patients aged 65 years or above, and thus also includes those with AF. So, strictly speaking, comparing this estimate with our study findings inherently underestimates the relative contribution of AF on mortality. Nevertheless, we observed a more than $50 \%$ higher IR of 6.7 per 100 person-years in our cohort of patients with $\mathrm{AF}$, illustrating the high risk of adverse events in patients with $\mathrm{AF}$ and indicating that these risks indeed are higher in patients with compared with those without AF.

\section{Clinical implication and future considerations}

Our results show that in the current era of highly effective anticoagulants, community-dwelling patients with $\mathrm{AF}$ have a greatly reduced risk of stroke, but still are at high risk of many adverse events. This stresses AF as a systemic condition and thus not solely a relatively benign cardiac arrhythmia, but both a cause and effect of multiple pathophysiological conditions. Its management, therefore, requires much more than solely the prevention of stroke prevention with anticoagulants and the control of rate and rhythm. Rather, it calls for integrated disease management, such as recommended by the European Society of Cardiology. ${ }^{15}$

Possibly using multidisciplinary teams, likely including at least community care specialists, cardiologists and hospitalists should aim to prevent all clinically relevant adverse outcomes for patients with AF. Examples include care as already implemented and evaluated in a hospital setting ${ }^{26}$ or currently under study in general practice. ${ }^{25}$

Identification of patients who may benefit most is an important step towards implementing such integrated care. Our study shows that the traditional well-known stroke risk factors may assist clinicians in this task. Targeting for instance the older patients with AF (eg, those $>80$ years old), those with heart failure and/or with concurrent vascular disease may efficiently prevent adverse outcomes. As an example, future studies may want to specifically target older patients with AF with either heart failure or those admitted to a coronary care unit with an acute coronary syndrome. Both situations are surely not hypothetical and in fact occur frequently in many cardiology clinics worldwide almost on a weekly basis. These patients should be flagged as 'high-risk' patients, calling for multidisciplinary teams delivering integrated care. Future research should evaluate such practices.

\section{CONCLUSION}

In anticoagulated community-dwelling patients with $\mathrm{AF}$, stroke risk is effectively reduced and thus fairly low, whereas risks of hospitalisation and mortality remain high, importantly mainly for non-cardiac causes. High age, heart failure and vascular disease consistently predict such outcomes and may be of value in identifying highrisk patients.

Contributors SvD contributed to the design of the study, performed data collection and data analysis, wrote the first manuscript draft and is accountable for all aspects of the work in ensuring that questions related to the accuracy or integrity of any 
part of the work are appropriately investigated and resolved. AT performed data collection and drafted the manuscript. FHR, AWH and KGMM interpreted the data and critically revised the manuscript. G-JG contributed to the design of the study, interpretation of the data and revised the manuscript. All authors approved of the version being published.

Funding The authors have not declared a specific grant for this research from any funding agency in the public, commercial or not-for-profit sectors.

Competing interests G-JG and FHR received an unrestricted institutional grant from Boehringer Ingelheim. KGMM and G-JG received a grant from The Netherlands Organization for Scientific Research (ZONMW 918.10.615 and 91208004, and ZONMW 016.166.030). All funding sources had no role in the design, conduct, analyses or reporting of the study or in the decision to submit the manuscript for publication. All other authors declared no conflict of interest.

\section{Patient consent Not obtained.}

Ethics approval As only deidentified data obtained from routine care were used, the Medical Ethics Committee of the University Medical Center Utrecht, the Netherlands, judged the CAFe study protocol as exempt from review as it was conducted outside the criteria for the Medical Research Involving Human Subjects Act (WMO).

Provenance and peer review Not commissioned; externally peer reviewed.

Data sharing statement For this study, we used data collected during routine clinical practice handled by a dedicated data infrastructure (Mondriaan). All relevant summary data are provided in the paper and its supplemental information. The committee of the network of general practices in which our study was conducted, is the formal owner of the research data ('The Julius General Practitioners' Network, JGPN, https://portal.juliuscentrum.nl/research/nl-nl/cohortsandprojects/ cohortsprojects/juliusgeneralpractitionersnetwork/jhn.aspx) and does not grant permission for the raw data to be made publicly available due to ethical and legal restrictions. All interested readers may request data without restriction from Dr Marlous Kortekaas (m.f.kortekaas@umcutrecht.nl), the study coordinator of the JGPN.

Open access This is an open access article distributed in accordance with the Creative Commons Attribution Non Commercial (CC BY-NC 4.0) license, which permits others to distribute, remix, adapt, build upon this work non-commercially, and license their derivative works on different terms, provided the original work is properly cited, appropriate credit is given, any changes made indicated, and the use is non-commercial. See: http://creativecommons.org/licenses/by-nc/4.0/.

\section{REFERENCES}

1. Go AS, Hylek EM, Phillips KA, et al. Prevalence of diagnosed atrial fibrillation in adults: national implications for rhythm management and stroke prevention: the AnTicoagulation and Risk Factors in Atrial Fibrillation (ATRIA) Study. JAMA 2001;285:2370-5.

2. Heeringa J, van der Kuip DA, Hofman A, et al. Prevalence, incidence and lifetime risk of atrial fibrillation: the Rotterdam study. Eur Heart $J$ 2006;27:949-53.

3. Krijthe BP, Kunst A, Benjamin EJ, et al. Projections on the number of individuals with atrial fibrillation in the European Union, from 2000 to 2060. Eur Heart J 2013;34:2746-51.

4. Lane DA, Skjøth F, Lip GYH, et al. Temporal trends in incidence, prevalence, and mortality of atrial fibrillation in primary care. J Am Heart Assoc 2017;6:e005155.

5. Colilla S, Crow A, Petkun W, et al. Estimates of current and future incidence and prevalence of atrial fibrillation in the U.S. adult population. Am J Cardiol 2013;112:1142-7.

6. Wolf PA, Abbott RD, Kannel WB. Atrial fibrillation as an independent risk factor for stroke: the Framingham Study. Stroke 1991;22:983-8.

7. Chimenti C, Russo MA, Carpi A, et al. Histological substrate of human atrial fibrillation. Biomed Pharmacother 2010;64:177-83.
8. Andrade J, Khairy P, Dobrev D, et al. The clinical profile and pathophysiology of atrial fibrillation: relationships among clinical features, epidemiology, and mechanisms. Circ Res 2014;114:1453-68.

9. Skalidis El, Hamilos MI, Karalis IK, et al. Isolated atrial microvascular dysfunction in patients with lone recurrent atrial fibrillation. J Am Coll Cardiol 2008;51:2053-7.

10. Geersing GJ, de Groot JA, Reitsma JB, et al. The impending epidemic of chronic cardiopulmonary disease and multimorbidity: the need for new research approaches to guide daily practice. Chest 2015;148:865-9.

11. Steinberg BA, Kim S, Fonarow GC, et al. Drivers of hospitalization for patients with atrial fibrillation: results from the outcomes Registry for Better Informed Treatment of Atrial Fibrillation (ORBIT-AF). Am Heart J 2014;167:735-42.

12. Chamberlain AM, Alonso A, Gersh BJ, et al. Multimorbidity and the risk of hospitalization and death in atrial fibrillation: a populationbased study. Am Heart J 2017;185:74-84.

13. Naccarelli GV, Johnston SS, Dalal M, et al. Rates and implications for hospitalization of patients $\geq 65$ years of age with atrial fibrillation/ flutter. Am J Cardiol 2012;109:543-9.

14. Odutayo A, Wong CX, Hsiao AJ, et al. Atrial fibrillation and risks of cardiovascular disease, renal disease, and death: systematic review and meta-analysis. BMJ 2016;354:i4482.

15. Kirchhof P, Benussi S, Kotecha D, et al. 2016 ESC Guidelines for the management of atrial fibrillation developed in collaboration with EACTS. Eur Heart J 2016;37:2893-962.

16. Gyh L, Nieuwlaat R, Pisters R, et al. Refining clinical risk stratification for predicting stroke and thromboembolism in atrial fibrillation using a novel risk factor-based approach: the euro heart survey on atrial fibrillation. Chest 2010;137:263-72.

17. Lamberts $\mathrm{H}$, Wood M. ICPC, international classification of primary care. World Organization of National Colleges A, and Academic Associations of General Practitioners/Family Physicians, et al. Oxford University Press, USA, 1987.

18. Kaatz S, Ahmad D, Spyropoulos AC, et al. Definition of clinically relevant non-major bleeding in studies of anticoagulants in atrial fibrillation and venous thromboembolic disease in non-surgical patients: communication from the SSC of the ISTH. J Thromb Haemost 2015;13:2119-26.

19. Christiansen CB, Olesen JB, Gislason G, et al. Cardiovascular and non-cardiovascular hospital admissions associated with atrial fibrillation: a Danish nationwide, retrospective cohort study. BMJ Open 2013;3:e001800.

20. Fumagalli S, Nieuwlaat R, Tarantini F, et al. Characteristics, management and prognosis of elderly patients in the Euro Heart Survey on atrial fibrillation. Aging Clin Exp Res 2012;24:517-23.

21. Kirchhof P, Schmalowsky J, Pittrow D, et al. Management of patients with atrial fibrillation by primary-care physicians in Germany: 1-year results of the ATRIUM registry. Clin Cardiol 2014;37:277-84.

22. Gómez-Outes A, Lagunar-Ruíz J, Terleira-Fernández Al, et al. Causes of death in anticoagulated patients with atrial fibrillation. J Am Coll Cardiol 2016;68:2508-21.

23. Lip GY, Laroche C, loachim PM, et al. Prognosis and treatment of atrial fibrillation patients by European cardiologists: one year followup of the EURObservational Research Programme-Atrial Fibrillation General Registry Pilot Phase (EORP-AF Pilot registry). Eur Heart J 2014;35:3365-76.

24. Pokorney SD, Piccini JP, Stevens SR, et al. Cause of death and predictors of all-cause mortality in anticoagulated patients with nonvalvular atrial fibrillation: data from rocket af. J Am Heart Assoc 2016;5:e002197.

25. van den Dries CJ, Oudega R, Elvan A, et al. Integrated management of atrial fibrillation including tailoring of anticoagulation in primary care: study design of the ALL-IN cluster randomised trial. BMJ Open 2017;7:e015510.

26. Gallagher $C$, Elliott $A D$, Wong $C X$, et al. Integrated care in atrial fibrillation: a systematic review and meta-analysis. Heart 2017;103:heartjnl-2016-310952. 